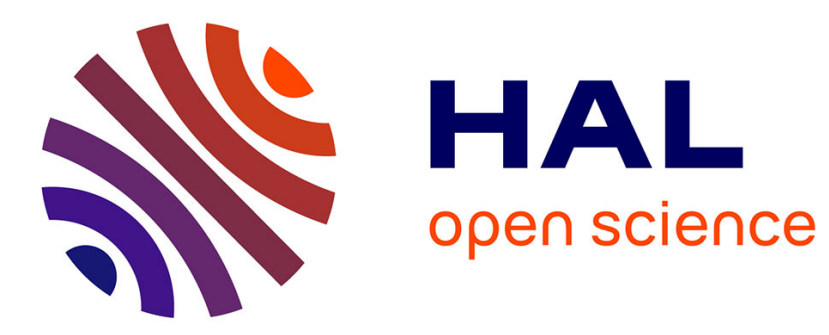

\title{
La contribution de l'économie expérimentale à l'analyse de l'efficacité des marchés
}

Stéphane Robin, Carine Staropoli

\section{To cite this version:}

Stéphane Robin, Carine Staropoli. La contribution de l'économie expérimentale à l'analyse de l'efficacité des marchés. Revue Française d'Economie, 2013, 28 (2), pp.91-120. halshs-00916937

\section{HAL Id: halshs-00916937 https://shs.hal.science/halshs-00916937}

Submitted on 11 Dec 2013

HAL is a multi-disciplinary open access archive for the deposit and dissemination of scientific research documents, whether they are published or not. The documents may come from teaching and research institutions in France or abroad, or from public or private research centers.
L'archive ouverte pluridisciplinaire HAL, est destinée au dépôt et à la diffusion de documents scientifiques de niveau recherche, publiés ou non, émanant des établissements d'enseignement et de recherche français ou étrangers, des laboratoires publics ou privés. 


\title{
La contribution de l'économie expérimentale à l'analyse de l'efficacité des marchés
}

\author{
Stéphane ROBIN \\ Carine STAROPOLI
}

Les premières expériences de marché réalisées par Vernon Smith [1962] ont mis en évidence l'importance des institutions de marché pour l'efficacité des échanges. Ces institutions organisent la communication d'information entre les acteurs du marché et structurent les incitations individuelles qui motivent l'échange. Elles ont une influence sur la dynamique des prix, le volume des transactions, l'efficacité allocative et informative du marché. Au cours des trente dernières années, l'économie expérimentale est devenue une méthode empirique utilisée pour le design de marché dans différents secteurs économiques. Le laboratoire est alors utilisé comme banc d'essai pour étudier les propriétés et mesurer les performances des institutions d'échange dans un environnement simple, contrôlé et reproductible. Dans cet article, nous illustrons l'apport de cette démarche à partir d'une revue des recherches conduites en laboratoire sur le design des marchés électriques.

The first market experiments conducted by Vernon Smith [1962] have highlighted the importance of market institutions for the efficiency of exchanges. These institutions organize the flows of information between market participants and incentivize individual decisions. They have an impact on prices' dynamics, trading volume, allocative and informational efficiency of the market. Over the past thirty years, experimental economics became an empirical method used to design market in various industries. The laboratory is used as a testbed to study the properties and measure the performances of any institutions in a simple, controlled and replicable environment. In this paper, we illustrate this approach by reviewing the main researches conducted in the lab on the design of electricity markets.

JEL Classification : C92, D02, D47

Mots clefs : Economie expérimentale, Design de marché, Institution de marché, Marchés de l'électricité

$\mathbf{U}_{\mathrm{n}}$ marché organisé fonctionne selon des règles qui lui sont propres. En économie expérimentale, on parle d'institution d'échange. Une institution est constituée de normes formelles et informelles fixant les procédures qui organisent l'échange sur le marché. Ces procédures établissent notamment l'identité des personnes qui peuvent intervenir sur un marché, les caractéristiques des produits échangés, la nature publique ou privée des informations sur ces caractéristiques, les modalités des soumissions de propositions d'achat et de vente, la détermination des prix et les contrats d'échange incluant le transfert de propriété. Pendant longtemps, les économistes se sont désintéressés de la question des règles d'échange. Cela s'explique d'une part par la quête d'une théorie générale des marchés applicable à toutes les formes de marché, d'autre part par le refus de considérer la pertinence de cette question. 
Dans cet article, nous nous appuyons sur une revue d'une sélection d'expériences de marché pour expliquer en quoi l'économie expérimentale permet de progresser dans l'analyse des institutions d'échange. En particulier nous montrons que l'économie expérimentale permet non seulement de tester ex post l'efficacité d'une institution de marché, mais qu'elle peut aussi participer ex ante à la conception d'institutions dans un contexte souvent complexe et peu stabilisé (les réformes concurrentielles dans les industries de réseaux par exemple).

Cet article met d'abord en évidence l'importance des institutions d'échange dans le champ de l'économie expérimentale (section 1). Nous présentons ensuite les bases méthodologiques des expériences (section 2). Nous expliquons enfin comment l'économie expérimentale est devenue une méthode empirique venant en appui aux décideurs chargés de la conception de marché, en nous référant aux travaux appliqués aux marchés de l'électricité (section 3).

\section{Au cour des expériences de marché : les institutions d'échange}

La question du design des institutions d'échange est au cœur des recherches en économie expérimentale de marché. Elle constitue même une des thématiques fondatrices de la méthode. De fait, c'est l'une des premières questions pratiques qui doit être traitée lors de la conception d'un marché en laboratoire. Quelles sont les règles qui vont encadrer les échanges entre les participants à l'expérience ? Le design des institutions d'échange recouvre plusieurs aspects : selon quelles règles les participants à l'expérience vont-ils échanger ? Comment les propositions d'échange doivent-elles être formulées et communiquées ? Si cela est autorisé, par quelle procédure ces propositions pourront-elles être révisées ? Est-ce que l'ensemble des participants au marché seront informés du prix de chaque transaction, ou bien cette information doit-elle être connue des seuls coéchangistes à l'exclusion des autres intervenants sur le marché ?

L'expérience conduite par Edward Chamberlin en 1948 est reconnue comme étant la première expérience de marché. Les résultats de cette expérience montrent des échanges peut efficace à des prix éloignés du prix d'équilibre concurrentielle (Chamberlin [1948]). L'expérience de Vernon Smith ${ }^{1}$ [1962], interpellé par les médiocres performances du marché concurrentiel observées par Chamberlin a approfondi la démarche initiée par Chamberlin. L'intuition de Smith est d'attribuer ce résultat à l'institution utilisée par Chamberlin. Chamberlin conduit son expérience en utilisant une négociation bilatérale décentralisée: vendeurs et acheteurs cherchent à parvenir à un échange en négociant en bilatéral, et en changeant de partenaire quand la négociation ne parvient pas à son terme. Smith souligne que cette procédure limite la diffusion de l'information sur le marché, et qu'elle rend plus difficile la convergence des prix altérant au final l'efficacité des échanges. Smith propose comme alternative d'utiliser la double enchère orale continue. Dans cette institution centralisée, l'information portée par les offres de vente, les offres d'achat et les transactions réalisées est rendue publique à l'ensemble des participants. Elle permet une convergence rapide vers l'équilibre concurrentiel. Ainsi, dès les premières expériences de marché réalisées dans des conditions contrôlées, il est clair que les institutions d'échange ne sont pas neutres pour ce qui est de la dynamique des marchés et de leur efficacité : elles ont une influence sur les comportements individuels, sur la formation des prix et sur la diffusion de l'information. Les travaux pionniers de Vernon Smith, puis ceux de Charles Plott au cours des années 1970 ont lancé un champ de recherche qui s'est imposé de façon durable. Au cours des dix dernières année, plus d'un article sur dix publié en économie expérimentale dans les principales revues économiques porte sur l'étude des institutions de marché stricto sensu, confirmant le dynamisme de ce champ de recherche (Noussair [2011]). Cela tient notamment au fait que les institutions de marché se renouvellent 
sans cesse à l'image des règles d'enchères inédites utilisées dans le cadre des réformes concurrentielles des industries d'infrastructures ou des places de marché sur internet $(B 2 B$ et $B 2 C)$ qui se sont multipliées ces dernières années.

Quels sont les objectifs poursuivis dans les expériences de marché ? En se référant à la typologie proposée par Roth [1995], on peut classer ces travaux en deux catégories. La première catégorie d'expériences de marché s'inscrit dans le cadre d'un "dialogue avec le théoricien". Il s'agit d'étudier en laboratoire des institutions de marché qui ont fait l'objet d'une analyse formelle. L'objectif est alors de mesurer la distance entre les stratégies optimales déterminées théoriquement pour l'institution considérée, et les comportements réels d'individus confrontés à cette institution en laboratoire. Une bonne illustration de ce type de recherche est constituée par les tests de la prédiction d'équivalence de revenu entre l'enchère sous pli au plus offrant au meilleur prix (ou enchère au premier prix) ${ }^{2}$ et l'enchère descendante (ou enchère hollandaise) ${ }^{3}$. Vickrey [1961] est le premier à avoir montré théoriquement que l'enchère au premier prix et l'enchère hollandaise permettent une allocation optimale identique pour le vendeur. Ce théorème connu comme le théorème d'équivalence du revenu a été invalidé en laboratoire par les expériences de Coppinger et al. [1980] et de Cox et al. [1982]. Ces expériences montrent en effet qu'en laboratoire, les prix de vente observés dans l'enchère au premier prix sont supérieurs à ceux obtenus pour l'enchère hollandaise. Cette différence est constatée indépendamment du nombre d'acheteurs en concurrence. Depuis, cette remise en cause du théorème d'équivalence a suscité de nombreux travaux théoriques et empiriques, parmi lesquelles de nouvelles expériences en laboratoire permettant de proposer une analyse comportementale de ces procédures de ventes (Kagel [1995]).

La seconde catégorie d'expériences de marché s'inscrit dans une démarche de "conseil aux décideurs" ${ }^{4}$. La grande innovation de cette branche de la littérature réside dans la définition d'un nouveau champ d'ingénierie : le design de marché. Le design de marché consiste à concevoir des institutions d'échange pour améliorer l'efficacité des marchés selon différents critères : revenus du vendeur ou de l'acheteur, efficacité allocative mais également stabilité des prix, réduction des coûts de transaction voire équité des allocations. Face à la difficulté ou l'impossibilité d'obtenir des données de terrain, l'objectif est de créer des données en laboratoire sur lesquelles s'appuyer pour obtenir des régularités empiriques qui permettent d'adresser des recommandations pratiques sur le choix des institutions d'échange et/ ou sur la révision des structures de marché. L'économie expérimentale participe alors directement au processus de conception d'institutions complexes qui doivent être créées ex-nihilo pour un objet et un contexte particulier. C'est le cas par exemple des enchères utilisées dans la vente des licences UMTS dans plusieurs pays européens dans les années 1980, mais aussi sur les bourses d'électricité ou dans le cas du mécanisme européen des droits d'émissions du $\mathrm{CO} 2$ (European Union Emissions Trading Scheme). Elle peut également résulter de la combinaison d'institutions plus simples dont les propriétés et les complémentarités, une fois qu'elles sont associées, ne sont pas connues théoriquement. L'économie expérimentale est alors utilisée comme banc d'essai pour ces nouvelles institutions de marché (Smith [2003]), la possibilité d'organiser un test grandeur nature n'étant pas envisageable compte tenu de l'ampleur des enjeux et de la lourdeur des dispositifs mobilisés. Là encore, les régularités empiriques obtenues en laboratoire sont utilisées pour mesurer l'efficacité des institutions testées, identifier et anticiper les stratégies possibles dans un environnement contrôlé et concevoir ex ante des « parades » aux éventuelles dysfonctionnements ou manipulations.

\section{Créer un marché en laboratoire : éléments méthodologiques}


Pour créer un marché en laboratoire, trois ingrédients sont nécessaires (Smith [1982b]) : l'environnement du marché, l'institution d'échange et les comportements des échangistes ${ }^{5}$. Nous reprenons ces trois ingrédients, en les illustrant dans le cadre d'une expérience de marché concurrentiel pour un bien homogène organisé selon les règles de la double enchère orale continue (double enchère ensuite dans le texte).

\section{L'environnement du marché}

L'environnement du marché comprend la liste des biens échangés sur le marché et la liste des participants autorisés à intervenir sur le marché. Chaque participant se caractérise par une compétence (vendeur, acheteur, trader), une fonction d'utilité ou de production, une dotation initiale en biens, en monnaie et en information. Ces caractéristiques sont en général des informations privées : chaque participant connaît la valeur pour lui des biens échangés mais il ne connaît la valeur attribuée à ces biens par les autres participants au marché. En construisant l'environnement du marché, le chercheur contrôle la structure du marché, les préférences des participants, la structure d'information et leur fonction respective sur le marché. Les fonctions d'utilités individuelles sont implémentées dans l'expérience via un mécanisme d'incitation qui associe la rémunération réelle de chaque participant à l'importance du surplus dans l'échange réalisé durant l'expérience (Smith [1976]). L'environnement de marché rassemble l'ensemble des éléments qui ne pourront être impactés par le marché au cours de l'expérience, ce qui n'exclue pas des évolutions possibles consécutives à l'acquisition d'informations au cours des échanges (information sur la valeur du bien ou la structure du marché par exemple).

Illustrons ce que l'on entend par ingrédients du marché avec l'exemple d'un marché concurrentiel avec un bien homogène pour lequel chaque agent attribue une valeur privée indépendante de celles des autres agents. On détermine dans un premier temps le nombre de vendeurs et le nombre d'acheteurs. Généralement, cinq vendeurs et cinq acheteurs sont un effectif suffisant pour obtenir un marché concurrentiel en laboratoire. Avant que ne s'engagent les premières négociations, chaque vendeur est informé de son coût (sa valeur limite à la vente, $c$ ) de chacune des unités qu'il peut proposer à la vente. Pour chaque unité vendue, le vendeur réalise un surplus égal à la différence entre le prix de vente $(p)$ et le coût de l'unité ( $p$ $-c$ ). Symétriquement, avant l'ouverture du marché, chaque acheteur est informé de sa valeur de reprise (sa valeur limite à l'achat, $x$ ) de chacune des unités qu'il pourra acheter sur le marché. Pour chaque unité achetée, l'acheteur réalisera un surplus dans l'échange qui sera égal à la différence entre la valeur de reprise $(x)$ et le prix d'achat $(p)$ soit $(x-p)$. Chaque transaction est donc un partage du surplus dans l'échange entre le vendeur et l'acheteur. Dans une expérience de marché, les participants savent qu'ils sont rémunérés en espèce à la fin de la session en fonction du surplus total réalisé sur le marché. Le choix des paramètres $(c, x)$ est effectué de manière à obtenir les fonctions agrégées de demande et d'offre désirées par le chercheur.

\section{L'institution de marché}

L'institution de marché se définie par l'ensemble des caractéristiques de la procédure d'échange. Dans la réalité, la procédure d'échange d'un marché organisé peut être particulièrement complexe ${ }^{6}$. De manière synthétique, une institution de marché comprend quatre composantes : les caractéristiques et la procédure de communication des offres d'échange sur le marché (offre de vente, offre d'achat, offre conditionnelle, etc.) ; le mécanisme d'enchère qui encadrent la procédure de communication; le mécanisme d'attribution qui détermine la façon dont le vainqueur est désigné ; la règle de paiement qui fixe le prix auquel l'échange s'effectue. 
La double enchère est sans doute l'institution la plus utilisée pour les expériences de marché. Deux raisons expliquent l'intérêt pour cette institution (Friedman [1993]). D'une part elle est la forme d'échange traditionnelle pour les biens homogènes (marché d'actions, marché de matières premières). D'autre part, cette institution est particulièrement efficace en termes de vitesse de convergence vers l'équilibre partiel concurrentiel, même quand le nombre de participants est réduit. Dans une double enchère, à tout moment et tant que le marché est ouvert, chaque vendeur a la possibilité de proposer une offre de vente ou d'accepter une offre d'achat déjà proposée par un acheteur. Symétriquement, chaque acheteur peut proposer une offre d'achat ou accepter une proposition de vente déjà formulée par un vendeur. Une offre de vente ou une offre d'achat consiste en un couple quantité/prix. L'acceptation d'une offre de vente ou d'une offre d'achat entraîne l'échange de tout ou partie de la quantité proposée au prix de l'offre. Une offre de vente reste active jusqu'à ce qu'elle trouve un preneur, ou qu'elle soit remplacée par une nouvelle offre de vente à un prix inférieur. Symétriquement, une offre d'achat reste active jusqu'à ce qu'elle trouve preneur, ou qu'elle soit remplacée par une nouvelle offre d'achat à un prix plus élevé. Aucun autre message n'est autorisé à l'exception d'une offre de vente, d'une offre d'achat, d'une acceptation d'offre de vente ou d'offre d'achat. L'une des caractéristiques principales de la double enchère, qui explique ses performances, est la centralisation et la diffusion de l'information : tout message envoyé sur le marché est diffusé simultanément à l'ensemble des participants. Cette condition explique en partie la convergence rapide des prix vers le prix d'équilibre concurrentiel et en conséquence l'efficacité des échanges en termes d'allocation.

\section{Le comportement des participants}

Si l'environnement et l'institution sont des variables contrôlées par le chercheur, les comportements des sujets qui participent à une expérience en laboratoire proviennent de l'observation et constituent à ce titre le "résultat » de l'expérience. Les participants à l'expérience de marché prennent leurs décisions en fonction des incitations introduites dans le design de l'expérience, de l'information dont ils disposent sur l'environnement du marché et en respectant le cadre de l'institution de marché. Les participants à l'expérience sont des individus réels dont le comportement s'éloigne généralement de celui de l'agent rationnel de la théorie. Par exemple, il n'est pas certain que les sujets perçoivent et mémorisent l'ensemble des informations échangées sur le marché. Il est aussi peu probable qu'ils utilisent de manière optimale cette information. À la différence d'une analyse théorique, l'expérience permet l'étude des propriétés d'une institution de marché avec des participants ayant une rationalité limitée. Dès lors, la formation des prix n'est pas un équilibre obtenu instantanément par le calcul rationnel de l'ensemble des participants, mais le résultat d'un processus dynamique dans lequel l'échange d'informations joue un rôle majeur (Smith [1982a]). La figure 1 représente le déroulement classique d'un marché de double enchère avec information privée sur la valeur des biens échangés ${ }^{7}$. Elle illustre le processus dynamique de convergence vers le prix d'équilibre dans une configuration de marché spécifique.

Figure 1

Convergence des prix dans un marché de double enchère 


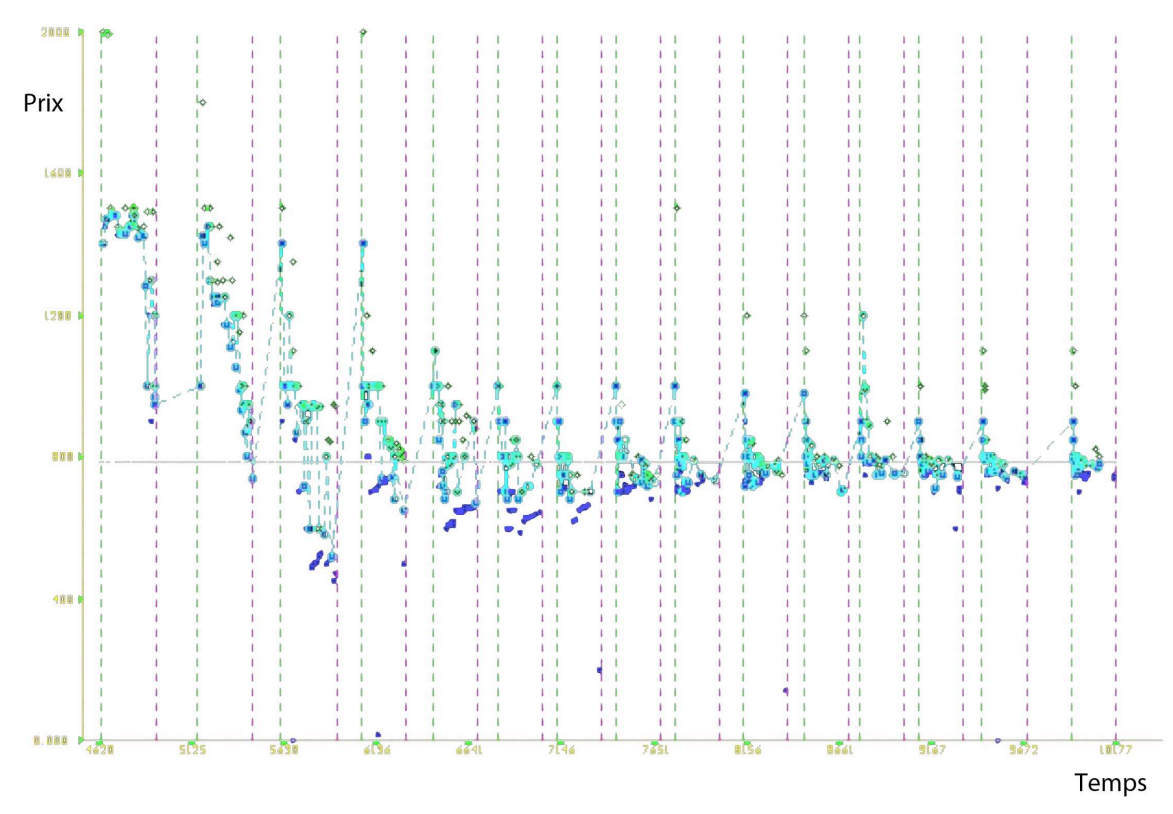

L'abscisse indique le temps, et l'ordonnée les prix. Les lignes verticales indiquent le début et la fin de chaque période d'échange. Ici l'expérience comporte 15 périodes d'échange. Les fonctions d'offre et de demande demeurent inchangées d'une période à l'autre ${ }^{8}$. Les points reliés indiquent les transactions effectives. Les offres d'achat sont représentées par des carrés, elles apparaissent au-dessous des transactions. Les offres de vente sont représentées par des losanges et elles apparaissent au-dessus des transactions. Enfin la droite horizontale correspond au prix d'équilibre concurrentiel, compte tenu des fonctions d'offre et de demande utilisées dans l'expérience. L'évolution des prix sur ce marché est exemplaire : les prix des échanges convergent vers le prix d'équilibre à l'intérieur d'une période d'échange à mesure que l'on se rapproche de la transaction marginale. Par ailleurs, les prix convergent vers le prix d'équilibre à mesure que l'on répète les périodes d'échange, et la variance des prix diminue. Parallèlement à la convergence des prix, l'efficacité des échanges s'accroit. Au bout de quelques périodes, le marché génère le surplus maximum qu'il est possible de réaliser, compte tenu des fonctions d'offre et de demande. Cette dynamique est le produit des décisions individuelles des participants au marché qui, partant d'une situation d'information limitée et incomplète, vont négocier en révisant leur croyance sur les prix praticables, à partir des informations sur les événements survenus sur le marché. L'institution de marché n'impose aucune restriction sur le volume des échanges ou sur le prix des transactions. Cependant, et de manière extrêmement robuste, les prix convergent toujours vers l'équilibre concurrentiel. La capacité prédictive du modèle d'équilibre partiel est avérée en laboratoire. Ce modèle élémentaire permet de déterminer l'équilibre du marché en prix et en quantité et l'évolution de cet équilibre en cas de choc sur l'offre et la demande (Jamison and Plott [1997]). Si le modèle de l'équilibre partiel permet bien de prédire l'évolution des prix, il ne dit rien sur la dynamique qui conduit à cette convergence. Cette dynamique est le produit d'un jeu complexe qui combine des stratégies de négociation des participants dans le cadre de l'institution de marché avec information croissante sur la valeur des biens échangés et apprentissage des participants sur ces valeurs ${ }^{9}$. L'étude de la dynamique des prix en laboratoire permet ainsi d'éclairer et de compléter un résultat théorique standard.

Si les expériences de marché ont permis d'étudier et de comparer les principales institutions de marché connues des économistes, elles ont aussi contribué à la conception de nouvelles 
institutions. La méthode expérimentale participe ainsi au design de nouvelles règles d'échange inédites.

\section{Les expériences de design de marché : une illustration par les marchés de l'électricité}

Les conditions d'émergence des marchés sont au cœur d'une controverse centrale en économie entre une vision libérale classique dans le prolongement des travaux d'Hayek qui voit le marché comme une création spontanée, et celle qui considère au contraire qu'un marché organisé est créé ex nihilo et qu'il faut s'intéresser au processus de création et aux composantes de ce marché. Cette vision est partagée par différents courants majeurs de l'économie dans le prolongement des travaux de Coase [1960] sur les externalités, l'économie des coûts de transaction et des droits de propriété, ceux d'Hurwicz [1973] pour l'approche du mechanism design ou encore ceux de Myerson sur le design d'enchère optimale dans une perspective théorique (Myerson, [1981]). L'intérêt pour cette question s'est accru avec la vague de réformes de libéralisation des industries de services publics qui ont eu lieu dans les années 1980 et 1990. L'éventualité d'un recours aux enchères est une question à traiter quand il s'agit d'introduire la concurrence dans des secteurs traditionnellement organisés comme des monopoles intégrés. L'utilisation d'une enchère pour l'attribution d'un droit de concession d'un service public selon le principe de la concurrence pour le marché (ou franchise biding) comme moyen de faire face au problème du monopole naturel local a été initialement théorisée par Chadwick [1859] puis par Demsetz [1968]. Cette pratique est aujourd'hui généralisée à de nombreux biens et services, qu'il s'agisse des services d'eau et d'assainissement, de la collecte et du traitement des déchets ou des concessions autoroutières. A ce sujet, les questions qui se posent sont les suivantes : faut-il utiliser une enchère ou bien d'autres dispositifs permettant de sélectionner un fournisseur ou un prestataire/ concessionnaire $^{10}$ ? Une fois que l'enchère est l'option choisie se pose alors un problème de mechanism design : il s'agit de choisir le format de l'enchère le plus approprié aux circonstances. Parmi les circonstances, il y a bien évidemment les spécificités de l'objet (ou des objets en cas de biens multiples), notamment le caractère commun ou non de la valeur du bien, mais aussi la nature combinatoire des différentes unités ou les conditions de production du bien. En termes de mechanism design, plus l'objet est spécifique, plus il faut agir au cas par cas, faire du sur mesure car les règles d'échange simples et standards ne peuvent pas être utilisées. Le design d'enchère n'est pas "one size fits all" (Kemperer [2002]). Par ailleurs, il faut tenir compte des structures de marché qui sont généralement le résultat d'une restructuration de l'industrie préalable à l'introduction de la concurrence, dans une activité longtemps organisée comme un monopole verticalement et horizontalement intégré (solution adoptée historiquement en raison de la situation de monopole naturel et de la nécessité de développer les infrastructures). Le lien entre les règles et les structures peut aller jusqu'à concevoir les règles avec pour objectif principal de minimiser les défaillances de marché résultant des structures mises en place.

Dans cette optique, l'économie expérimentale constitue un outil précieux pour étudier les propriétés d'efficacité des règles d'échange, en complément d'autres approches théoriques et empiriques. Les expériences permettent de mettre en évidence des défaillances de marché et des comportements stratégiques qui reposent sur la manipulation des règles de marché en fonction des structures. En effet, si des sujets sont en mesure, dans un environnement simplifié et contrôlé, de manipuler les règles de marché dans leur propre intérêt au détriment de l'efficacité du marché (mettant en évidence les faiblesses et le manque de robustesse de l'institution considérée), tout porte à croire que la transposition dans la réalité se heurtera à des difficultés encore plus importantes. Le tableau suivant présente une sélection d'expériences de 
marché qui ont été conduites dans des domaines et des contextes variés, et qui ont permis de mettre en évidence des problématiques propres aux différentes industries, et d'apporter des solutions de design adaptées, le cas échéant.

\begin{tabular}{|c|c|c|c|c|}
\hline Secteurs & Marché & Article & Problématique & Solution préconisée \\
\hline Transport aérien & $\begin{array}{l}\text { Allocation des slots } \\
\text { d'atterrissage et de décollage }\end{array}$ & Rassenti et al. [1982] & $\begin{array}{l}\text { Allocation simultanée de } \\
\text { biens complémentaires }\end{array}$ & $\begin{array}{l}\text { Enchère combinatoire } \\
\text { sous pli }\end{array}$ \\
\hline Télécommunication & $\begin{array}{l}\text { Allocation des spectres de } \\
\text { fréquences hertziennes }\end{array}$ & Bank et al. [2003] & $\begin{array}{l}\text { Allocation simultanée de } \\
\text { biens complémentaires }\end{array}$ & $\begin{array}{l}\text { Enchère combinatoire en } \\
\text { plusieurs étapes }\end{array}$ \\
\hline Marché internet & $\begin{array}{l}\text { Vente en ligne de type CtoC } \\
\text { ou BtoC }\end{array}$ & Ariely et al. [2005] & $\begin{array}{l}\text { Inefficacité liée aux } \\
\text { soumissions de dernière } \\
\text { minute }\end{array}$ & $\begin{array}{l}\text { Extension du la durée de } \\
\text { la vente automatique si } \\
\text { soumission de dernière } \\
\text { minute }\end{array}$ \\
\hline Transport ferroviaire & $\begin{array}{l}\text { Attribution du droit de faire } \\
\text { transiter un train sur le réseau }\end{array}$ & Brewer et Plott [1996] & $\begin{array}{l}\text { Allocation simultanée de } \\
\text { combinaisons de produits à la } \\
\text { fois substituables et } \\
\text { complémentaires } \\
\text { Prise en compte des priorités } \\
\text { entre types de trains }\end{array}$ & $\begin{array}{l}\text { Enchère continue à prix } \\
\text { constant sous contrainte } \\
\text { des conflits entre } \\
\text { allocations incompatibles } \\
\text { (binary conflict ascending } \\
\text { price mechanism) }\end{array}$ \\
\hline Transport du gaz & $\begin{array}{l}\text { Attribution du droit de faire } \\
\text { transiter un flux sur un réseau } \\
\text { de pipelines }\end{array}$ & Rassenti et al.[1994] & $\begin{array}{l}\text { Allocation simultanée de } \\
\text { combinaisons de produits à la } \\
\text { fois substituables et } \\
\text { complémentaires. }\end{array}$ & $\begin{array}{l}\text { Double enchère à prix } \\
\text { continu sous contrainte du } \\
\text { réseau de pipelines }\end{array}$ \\
\hline Logements & $\begin{array}{l}\text { Attribution de logements aux } \\
\text { étudiants }\end{array}$ & $\begin{array}{l}\text { Chen et Sönmez } \\
\text { [2002] }\end{array}$ & $\begin{array}{l}\text { Allocation de produits } \\
\text { indivisibles à des agents dont } \\
\text { certains ont déjà un produit } \\
\text { attribué antérieurement }\end{array}$ & $\begin{array}{l}\text { Mécanisme d'assortiment } \\
\text { original : le top trading } \\
\text { cycles mechanism }\end{array}$ \\
\hline Agriculture & $\begin{array}{l}\text { Attribution des droits à } \\
\text { irriguer en période de } \\
\text { sécheresse }\end{array}$ & $\begin{array}{l}\text { Cummings et al. } \\
\text { [2004] }\end{array}$ & $\begin{array}{l}\text { Allocation de produits à } \\
\text { valeurs communes et privées } \\
\text { non connus avec risque de } \\
\text { collusion }\end{array}$ & $\begin{array}{l}\text { Enchère sous pli itérative } \\
\text { à prix discriminants }\end{array}$ \\
\hline
\end{tabular}

Dans cet article, nous avons choisi d'illustrer l'apport de l'économie expérimentale dans le domaine du design de marché dans le contexte des réformes de libéralisation de l'industrie électrique. En raison des spécificités de l'électricité, la mise en œuvre de marchés électriques organisés soulève de nombreux problèmes génériques que l'on peut retrouver dans d'autres secteurs. Toutefois, mis bout à bout, ces problèmes font du design des marchés électriques une véritable gageure. Les économistes se sont intéressés à ce sujet avec différentes optiques qu'il s'agisse des travaux fondateurs de Joskow et Schmalensee [1983] sur la dérégulation du secteur dans une perspective d'économie industrielle et institutionnelle, ou de ceux Wilson [2002], de Stoft [2002] dans une perspective de mechanism design appliqué au secteur électrique.

L'économie expérimentale dans le prolongement des travaux pionniers de Vernon Smith dès les années $1980^{11}$ a contribué à la mise en œuvre des réformes dans différents pays. Avant de passer en revue différentes recherches conduites dans ce champ, il est nécessaire de passer par une présentation des principales caractéristiques de l'électricité ayant un impact sur les problématiques de design de marché. 


\section{Spécificités de l'électricité}

L'électricité n'est pas un bien standard (une commodité). Elle cumule plusieurs caractéristiques sources de complexité du point de vue de l'économiste. L'électricité est un bien difficilement stockable. Sa demande est inélastique sur le court et le très court terme, variable sur plusieurs périodicités (jour, semaine et année), et elle est en partie incertaine (soumise à différents aléas de nature climatique, comportementale et technologique). Pour éviter tout risque d'effondrement du réseau ${ }^{12}$, il est nécessaire que la production puisse s'ajuster à la demande en temps réel. Or la production ${ }^{13}$ d'une part, et le transport et la distribution $^{14}$ d'autre part, présentent certaines rigidités qui contraignent l'équilibre offredemande en temps réel. Dans certaines situations, l'équilibre offre demande est obtenu au prix du délestage d'une partie du réseau ce qui entraine des coupures localisées. Une transaction entre un vendeur et un acheteur d'électricité se traduit par un droit d'injecter (de soutirer) pour le vendeur (l'acheteur) de l'énergie sur un point du réseau (Wilson [2002]). Optimiser l'utilisation de ces différents moyens de production pour répondre en temps réel à une demande variable et incertaine est donc un problème de design complexe. Cela est d'autant plus vrai quand il doit être traité de manière décentralisée par des acteurs indépendants via les marchés selon une logique économique déconnectée des flux physiques.

\section{Le design des marchés électriques : problématique et expériences}

L'utilisation de marchés organisés dans le secteur de l'électricité vise à atteindre deux objectifs. Dans le cours terme, il s'agit d'assurer l'efficacité productive et allocative du système : garantir, d'une part, l'équilibre offre-demande en temps réel en minimisant le coût de production et, d'autre part, aboutir à la situation pour laquelle le bénéfice marginal des consommateurs est égal au coût marginal de production. Dans le long terme, les marchés doivent permettre de rémunérer les différents acteurs de la filière (producteurs, opérateurs des réseaux de transport et de distribution et fournisseurs) et d'orienter leur investissement de manière à maintenir la sécurité et la sûreté du système électrique et assurer sa pérennité.

L'observation des designs de marché électrique existants montre qu'il existe une grande variété de solutions mises en œuvre pour atteindre ces objectifs. Les différences portent les différents ingrédients des marchés, à commencer par les produits échangés sur ces marchés : l'énergie physique (en $\mathrm{kWh}$ ), l'attribution des droits d'utiliser les lignes de haute tension ou encore le mode de rémunération des services auxiliaires nécessaires à la fiabilité du réseau (principalement des réserves de productions mobilisables en quelques secondes en cas de déséquilibre entre les quantités injectées et soutirées) ${ }^{15}$. La diversité des designs porte aussi la définition spatio-temporelle des marchés organisés. Par exemple, EpexSpot est la bourse unique des marchés spot français, allemand, autrichien et suisse ce qui illustre la volonté d'intégrer, dans la mesure du possible, les différents marchés européens en fonction des capacités d'interconnexion entre les pays. EpexSpot propose différentes places de marché, journaliers, infra-journaliers et en continu, pour permettre aux acteurs de modifier leur position à mesure que l'incertitude se réduit sur le condition de l'équilibre offre-demande. Enfin les marchés se différencient selon les produits qui sont échangés (types de contrats avec livraison physiques), et les institutions d'échange utilisées avec des règles de soumission des offres, de fixation des prix ou d'allocations finales variées.

Ainsi, il existe une multitude de solutions possibles pour organiser les marchés de l'électricité. Il est souvent difficile d'établir l'intérêt et les limites de chaque alternative dans l'absolu et relativement. Dès la fin des années 1980, l'économie expérimentale est utilisée comme une méthode d'évaluation et de comparaison des designs de marché dans ce secteur. Les premières expériences de marché visaient à estimer la faisabilité d'un abandon du système de monopole régulé qui organisait de manière centralisée la production, le transport et la distribution de 
l'électricité. Sollicitée par le régulateur du secteur électrique d'Arizona (Arizona Corporation Commission) en 1984, l'équipe de Vernon Smith à l'Université d'Arizona réalise les premières expériences de marché en laboratoire sur ce secteur grâce à l'utilisation de smart market ${ }^{16}$.

Ces travaux précurseurs ont non seulement permis de montrer qu'il était possible de reproduire en laboratoire une situation aussi complexe que celle d'un marché électrique, mais en plus, ils ont pu faire un certain nombre de recommandations concernant l'architecture du marché notamment la séparation verticale des infrastructures de transport et de distribution et de la production, les mécanismes du marché de l'énergie et l'allocation des droits de propriété sur le réseau (Rassenti et Smith [1986]). Dans un premier temps, leurs propositions ont été accueillies avec beaucoup de réticences par la Commission, mais elles ont permis de lancer un agenda de recherche qui a donné lieu à des prolongements dans plusieurs directions que nous allons passer en revue.

\section{Limiter le pouvoir de marché des producteurs sur le marché de l'énergie}

Les structures de marchés dans le secteur électrique sont historiquement centralisées horizontalement et verticalement en raison des économies d'échelles en production et de la présence d'un monopole naturel au niveau des infrastructures de transport et de distribution. Malgré les restructurations qui accompagnent la libéralisation, les marchés de l'énergie sont caractérisés par la présence de pouvoirs de marchés des producteurs qui réduisent l'efficacité de la concurrence. Les expériences en laboratoire ont montré qu'il est possible de réduire l'impact de cette situation en choisissant l'institution de marché adaptée. Denton et al. [2001] ont étudié en laboratoire deux institutions alternatives : une enchère bilatérale sous pli et une double enchère continue à prix uniforme. Dans cette institution de marché, les producteurs peuvent, en continu, soumettre des offres d'échange et les réviser jusqu'à ce que l'offre et la demande s'équilibrent. L'ensemble des échanges s'effectue alors au prix d'équilibre. Deux structures concurrentielles sont étudiées, une première structure avec 3 producteurs (marché concentré), une seconde avec 6 producteurs (marché concurrentiel). Étonnamment, c'est avec l'enchère sous pli que le pouvoir de marché produit le moins de perte en efficacité. Il n'y a pas de différences observées entre la configuration à 3 producteurs et celle à 6 producteurs. La double enchère à prix uniforme est moins efficace, en particulier dans configuration à 3 producteurs. Cette étude montre que l'exercice d'un pouvoir marché est ici fortement dépendant du choix des règles d'échange. La possibilité offerte par la double enchère continue d'observer les offres des autres producteurs et de réviser ses positions en conséquence facilite la manipulation des prix sur le marché et l'exercice d'un pouvoir de marché.

L'article de Brandts et al. [2013] s'intéresse au pouvoir de marché des producteurs pivots sujet d'intérêt pour les autorités de régulation. Un producteur est qualifié de pivot quand le retrait de son offre réduit l'offre agrégée du marché à un niveau inférieur à la demande ce qui perturbe l'équilibre offre demande. Ces producteurs contribuent à la l'augmentation des prix et à l'inefficacité des marchés. L'étude montre que les producteurs pivots ont effectivement le pouvoir de faire monter les prix sur le marché. Même lorsque les capacité de production agrégée sont excédentaire par rapport à la demande, la présence de producteur pivot empêche le marché de converger vers le prix concurrentiel. L'expérience permet de comparer la performance de deux modèles d'estimation du pouvoir de marché des producteurs pivots qui peuvent être utilisé pour estimer la présence de pouvoir de marché sur le terrain.

Une autre manière de limiter le pouvoir de marché des producteurs est d'introduire une participation active de la demande sur le marché, ce qui est loin d'être intuitif en raison du caractère inélastique à court terme de la demande d'électricité. L'étude de Rassenti et al. [2003] montre qu'en laboratoire, la participation de la demande sous la forme d'une enchère de demande permet de neutraliser l'exercice de pouvoirs de marché des producteurs et de 
réduire significativement la volatilité des prix en période de pointe. L'article de Jullien et al. [2008] étudie en laboratoire les conditions d'efficacité d'un mécanisme permettant aux consommateurs de réduire leur consommation en réaction aux prix de marché, on parle alors d'effacement de la demande. Les résultats montrent que l'introduction d'un tel mécanisme conduit à une réduction significative des prix de marché en périodes de pointe (pic de demande) et qu'il empêche les producteurs d'exercer leur pouvoir de marché en périodes de demande intermédiaire. Cependant, l'étude montre qu'en environnement constant, après répétition des périodes d'échanges, les producteurs trouvent des stratégies pour contrer les effets des effacements de la demande sur les prix. Ces résultats conduisent à tempérer l'enthousiasme de ceux qui soutiennent qu'un marché électrique devrait se rapprocher du fonctionnement d'un marché de commodité standard, c'est à dire adopter des règles génériques. Toutefois, les mécanismes de contrôle de la demande dans le contexte de la transition énergétique et des politiques de maitrise de la demande devraient connaître dans un futur proche un regain d'intérêt avec le développement des réseaux intelligents (smart grids) et des compteurs intelligents (Sioshansi [2012]) dont l'objectif est précisément de proposer des solutions techniques permettant d'accroitre la réactivité de la demande au prix ${ }^{18}$.

\section{Allouer les capacités de transport}

L'allocation efficace des capacités de transport sur un réseau électrique est une question majeure pour l'efficacité du système électrique d'une part et pour celui des échanges sur les marchés de l'énergie d'autre part. Ainsi, certaines situations de pouvoirs de marché sont liées aux caractéristiques du réseau de transport : un producteur injectant de l'énergie dans une zone isolée du reste du réseau pourra tirer avantage de cette situation et imposer des prix plus élevés. On parle alors de rente de congestion. L'économie expérimentale permet de traiter, tout en la simplifiant, la complexité du système électrique, là où les modèles analytiques sont limités. Il est ainsi possible de faire des expériences de marché avec des réseaux maillés de tailles significatives ${ }^{18}$ tout en intégrant les contraintes de réseau qui découlent des spécificités physiques de l'électricité : flux de bouclage, congestions, nature stochastique de la charge sur les lignes, équilibre en temps réel de l'offre et de la demande, variabilité localisée des pertes de transport. Dans le cadre d'un réseau élémentaire comportant trois nœuds répartis sur une ligne de transport, Backerman et al. [2000] analysent la création et le partage de la rente de congestion en fonction du type de tarification des capacités de transport utilisées. Cette étude montre que les producteurs peuvent capter la rente de congestion au détriment des gestionnaires de transport et des acheteurs. L'étude de Jullien et al. [2012] compare deux institutions de marché permettant l'allocation de capacités de transport d'électricité : les enchères implicites et les enchères explicites coordonnées. Les enchères implicites sont actuellement utilisées dans les pays d'Europe du Nord. Dans ce mécanisme, l'énergie et les capacités de transport sont traitées simultanément. Lorsqu'une congestion apparait, son prix i.e. son coût ou sa rémunération - correspond à la différence entre les prix de l'énergie de chaque côté de l'interconnexion congestionnée. Dans une enchère explicite coordonnée, le marché du transport est organisé en amont du marché de l'énergie.

Figure 2

Capacité du réseau et positionnement des concurrents (Jullien et al. [2012]) 


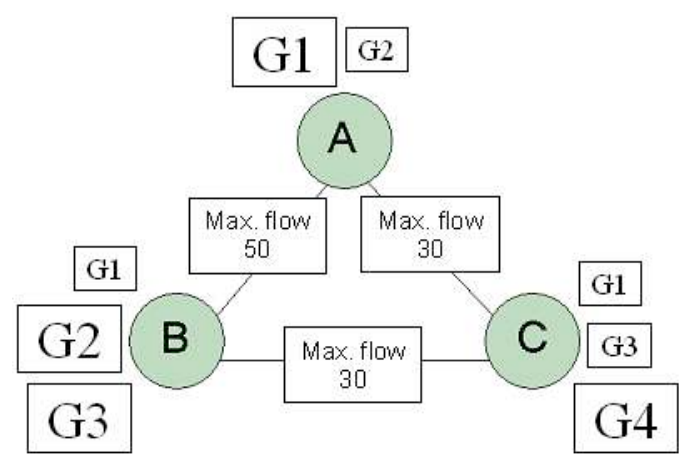

Le design de l'expérience repose sur une maquette représentée sur la figure 2. Elle modélise un réseau électrique élémentaire comportant trois zones $(A, B$ et $C$ ) séparées par des lignes de transport ayant des capacités de transit limitées. Les concurrents $(\mathrm{G} 1, \ldots, \mathrm{G} 4)$ sont des producteurs positionnés sur les différents nœuds du réseau avec un pouvoir de marché sur l'un de ces nœuds. L'enjeu de cette étude est d'évaluer si les deux institutions de marché permettent ou non d'allouer de manière efficace les capacités de transport limitées du réseau, tout en contrôlant l'exercice de pouvoir de marché et les comportements stratégiques inefficaces sur le marché de l'énergie. Les résultats montrent que l'enchère explicite coordonnée (solution envisagée au niveau européen) n'est pas efficace, à la fois en termes de surplus créé dans l'échange et d'allocation des capacités de transport. L'enchère implicite est nettement plus performante. Peut-on expliquer l'inefficacité de l'enchère explicite coordonnée ? Dans l'environnement simplifié créé en laboratoire, les participants anticipent mal les prix, les effets de l'offre et ceux de la demande sur le marché de l'énergie. En conséquence, ils prennent des décisions inefficaces sur le marché des capacités de transport sur lequel ils doivent s'engager ex-ante. L'inefficacité observée pour l'enchère explicite coordonnée vient des caractéristiques propres au mécanisme. Par principe, les capacités acquises sur le marché du transport doivent être entièrement utilisées à chaque période. Cette règle crée de l'irréversibilité dans les décisions d'achat de capacités de transport, elle accentue les erreurs d'anticipation et elle perturbe la formation des prix sur ce marché. Ce résultat incite à envisager avec prudence la transposition dans la réalité de ce mécanisme dans un contexte forcément plus complexe notamment au niveau des anticipations.

\section{Améliorer l'efficacité des échanges sur les marchés de l'énergie à court terme}

Comparativement aux autres secteurs, il existe de nombreuses études expérimentales consacrées aux institutions de marché utilisées sur les marchés électriques de l'énergie. Hahn et Boening [1990] étudient deux règles de fixation des prix dans le cadre d'un marché électrique. Le première est une règle couramment utilisée : la règle de split-saving. Pour une offre d'achat appariée à une offre de vente, le prix d'échange est fixé à la moyenne des prix des deux offres. Cette règle est largement diffusée mais ses propriétés sont peu connues dès lors que les offres d'échange sur le marché n'expriment pas les prix limites à la vente et à l'achat des opérateurs. Hahn et Boening [1990] comparent cette règle à une enchère sous pli à prix uniforme. Ils observent que la règle de split-saving est moins efficace qu'une enchère sous pli à prix uniforme et qu'elle conduit des prix plus éloignés du prix d'équilibre concurrentiel. Ils observent aussi que pour les deux institutions testées, les offres d'échange ne révèlent pas les valeurs réelles qu'attribuent les participants au produit échangé. Toujours dans le cadre d'un marché électrique de l'énergie, Bernard et al. [1998] s'intéressent à deux règles de fixation des prix dans le cadre d'une enchère unilatérale sous pli à prix uniforme : l'échange au prix de la première offre refusée et l'échange au prix de la dernière offre acceptée. Les résultats obtenus montrent que la seconde procédure permet des échanges plus efficaces à des prix plus proches du prix concurrentiel. Ils mettent aussi en évidence que la structure 
concurrentielle domine l'influence des règles de fixation de prix dans une enchère unilatérale. Partant de ce constat, les auteurs préconisent l'introduction d'une enchère bilatérale avec participation de la demande.

\section{Inciter les producteurs à investir dans des centrales de pointe ${ }^{19}$}

La sécurité d'un système électrique repose sur l'existence de moyens de production pouvant être mobilisés en cas de pic de la demande. L'investissement sur ces centrales de pointe est confronté à un problème structurel de rentabilité mis en évidence par Joskow [2006]. D'une part, leur taux d'utilisation est par définition très faible. D'autre part, les prix qui reflèteraient la valeur de la production en période de pic de demande et qui pourraient assurer la rentabilité de ces équipements dépasse la limite de prix souvent imposée sur les marchés par le régulateur. Les marchés de capacités sont des mécanismes qui visent à assurer la rentabilité de ce type de centrales. Ils fournissent les incitations pour la construction de ces équipements nécessaires à la sécurité du système électrique. Jullien et al. [2009] étudie un marché de capacités à termes conçu pour pallier aux défaillances des mécanismes existants. Dans ce mécanisme, un marché des capacités est organisé jusqu'à trois ans en avance (délais nécessaire pour construire une centrale de pointe) pour des engagements à fournir de l'électricité à un prix donné durant les périodes de pic de demande à venir. Les producteurs participants à ce marché soumettent des offres qui comportent le volume qu'ils s'engagent à fournir si cela est requis, et le prix demandé pour la rémunération annuelle de cet engagement. Un producteur retenu sur le marché des capacités à terme recevra cette rémunération pendant la durée de son engagement. En contrepartie, durant les pics de demande d'électricité, il devra impérativement proposer sur le marché spot les volumes attendus au prix fixé à l'avance. Ces capacités de production offertes à la vente à un prix convenu permettent d'assurer l'équilibre offre-demande, de réduire le niveau et la volatilité des prix. La comparaison de ce mécanisme avec un design sans marché de capacités à terme montre que ce dernier permet effectivement d'améliorer l'efficacité allocative en assurant un niveau d'investissement efficace, et en limitant le niveau des prix sur le marché spot en période de pointe. Ce type de mécanisme vient compléter les modes de rémunération classique par les marchés de l'électricité en résolvant une défaillance qui compromettait la viabilité du système. En France la loi NOME voté en 2010 a prévu la mise en place d'un marché de capacité à terme. Cependant les conditions de sa mise en œuvre ont suscité de nombreux débat et la mise en place de marché est maintenant prévue pour 2015.

\section{Conclusion}

Le but recherché dans le design des mécanismes économiques est d'aligner les incitations individuelles avec les objectifs souhaitables d'un point de vue collectif. Les institutions de marché sont des mécanismes de coordination entre des agents économiques qui prennent des décisions de manière décentralisée, sur la base d'informations privées. Le design de marché vise à concevoir les institutions d'échange afin de favoriser l'efficacité des échanges, l'agrégation et la communication d'information ou encore pour réduire les coûts de transaction. La performance d'une institution de marché peut être évaluée de manière théorique. Cependant ces analyses reposent sur un ensemble d'hypothèses comportementales souvent éloignées des comportements réels des agents économiques.

L'économie expérimentale est une méthode empirique qui permet de vérifier les propriétés attendues d'une institution de marché dans un environnement simplifié, contrôlé et reproductible. Il s'agit de construire en laboratoire une maquette simplifiée de la réalité capturant l'ensemble des variables importantes pour la question traitée, et d'analyser le comportement d'individus réels dans cette maquette. Ces expériences de marché conduites en laboratoire peuvent être comparées à un banc d'essai pour les mécanismes économiques. Elles 
permettent, sans les coûts d'un déploiement complet sur le terrain, de tester de nouvelles règles d'échange dans un environnement contrôlé avec des agents économiques réels prenant des décisions ayant des conséquences sur leur bien-être.

L'économie expérimentale constitue aussi un outil d'investigation intéressant pour étudier des institutions dont l'étude est hors de portée des possibilités offerte par l'analyse théorique. Les expériences de marché permettent alors d'observer la dynamique de l'institution, ses propriétés, mais aussi ses limites et ses défaillances. Les récentes évolutions liées à la libéralisation des industries d'infrastructures comme l'industrie électrique ou le développement spectaculaire des marchés virtuels ont fourni des champs d'applications qui ont, en partie, motivé ce développement. Depuis la création des marchés électriques, de nombreuses études ont été conduites en laboratoire sur des enjeux variés (tarification, enchères, structures de marché). Certaines - on pense notamment aux travaux de Vernon Smith - ont été décisives dans les choix des autorités en charge de la mise en œuvre de ces marchés. Dans un futur proche, on peut penser que les enjeux environnementaux couplés aux innovations technologiques des réseaux intelligent ouvrent de nouveaux champs d'investigations qui vont couvrir le niveau des marchés de détail jusqu'ici ignoré car toujours régulés. Il s'agit de concevoir des modes de tarification incitative ou des vecteurs d'information pour parvenir à une maitrise partielle de la demande. L'enjeux est, dans la perspective de la transition énergétique, de lisser la consommation le long de la journée, de réduire la consommation totale mais aussi de pouvoir faire face à l'aléa croissant qui pèse sur l'offre d'électricité avec l'intégration des énergies renouvelables. Ce champ appelle à développer de nouvelles méthodes expérimentales combinant les études en laboratoire et des expériences sur le terrain en utilisant les nouvelles technologies de l'information et de la communication.

Les auteurs remercient Olivier L'Haridon pour son soutien et ses précieux commentaires sur la version préliminaire de cet article ainsi que le rapporteur de la revue pour ses commentaires et suggestions.

Stéphane Robin est chargé de recherche au GATE LSE. University of Lyon, 69007 Lyon, France; CNRS, GATE Lyon St Etienne 69130 Ecully, France. robin@gate.cnrs.fr

Carine Staropoli est chercheur au Centre d'Economie de la Sorbonne, Université PanthéonSorbonne, Paris, France et Directrice scientifique adjointe de la Chaire Economie des Partenariats Public Privé (IAE, Paris). carine.staropoli@univ-paris1.fr

\section{Notes}

1. Le travail de Vernon Smith sur l'étude des institutions d'échange a été récompensé en 2002 par l'attribution du prix de la Banque Royale de Suède en sciences économiques en mémoire d'Alfred Nobel "pour avoir instauré la démarche expérimentale en laboratoire comme un outil d'analyse empirique de l'économique, en particulier dans l'étude des mécanismes de marché alternatifs ».

2. Dans une enchère au premier prix, plusieurs acheteurs sont en concurrence pour l'achat d'un bien. Chacun soumet une offre d'achat sous pli, simultanément et sans communiquer avec les autres enchérisseurs. Les offres sont collectées par le commissaire priseur, l'acheteur 
sélectionné est celui qui fait la proposition de prix la plus élevée. Cet acheteur doit payer le montant de son offre pour acquérir le bien.

3. Cette procédure est utilisée pour la vente de fleurs en gros en Hollande : une horloge décrémente des prix face aux acheteurs potentiels réunis dans un même lieu. Le premier acheteur qui arrête l'horloge remporte le lot et paye le dernier prix indiqué par l'horloge.

4. Littéralement "Whispering in the Ears of Princes", (Roth [1995]).

5. Les techniques et le matériel nécessaire à la conception d'un marché expérimental sont présentés de façon très complète par Plott et Gray [1990], Davis et Holt [1993], ainsi que par Friedman et Sunder [1994].

6. À titre d'illustration, on pourra se reporter à la documentation sur les règles de fonctionnement de la bourse de l'électricité EpexSpot (http ://www.epexspot.com).

7. Les données utilisées pour ce graphique sont issues d'une session expérimentale présentée dans l'article de Noussair et al. [1998].

8. En laboratoire, cela se traduit par le fait qu'au début de chaque période, les acheteurs retrouvent les mêmes valeurs de reprise et les vendeurs les mêmes coûts qu'à la période précédente.

9. Pour des tentatives de modélisation de cette dynamique de convergence, on pourra se référer à l'ouvrage collectif coordonné par Daniel Friedman et John Rust consacré aux marchés de double enchère (Friedman et Rust [1993]).

10. Parmi les autres dispositifs envisageables on peut citer la négociation bilatérale qui n'implique pas forcément de mise en concurrence, les procédures administratives (par exemple les règles de prorata ou de premier arrivé-premier servi) ou le concours de beauté.

11. Vernon Smith et Stephen Rassenti ont commencé à travailler sur le design des marchés de l'énergie dès 1986 (Rassenti et Smith [1986]). Ces travaux s'effectuent dans le cadre de projets mandatés par des autorités en charge de la dérégulation en Nouvelle Zélande et en Australie (Rassenti et al. [2002]).

12. Un effondrement du réseau vient d'une mise hors tension en cascade des lignes de transport et de distribution. Cela se traduit par une panne de courant (blackout) de plus ou moins grande ampleur. La panne la plus importante de l'histoire au sens du nombre d'individus concernés (670 millions d'usagers) est celle qui frappa d'Inde en 2012. L'Europe ou l'Amérique du Nord ont aussi connu dans les années récentes plusieurs pannes d'envergure qui ont mis en évidence des failles des systèmes électriques.

13. La production d'électricité repose sur un mix énergétique composé d'un ensemble de technologies et de combustibles (hydraulique, nucléaire, gaz, charbon, fuel, renouvelable etc.) permettant la production d'énergie avec des coûts de production et des conditions d'exploitation très différents. Ainsi les centrales nucléaires se caractérisent par de faibles coûts de production mais par une faible capacité d'adaptation à court terme (il n'est pas possible d'interrompre en temps réel la production d'une centrale nucléaire ni de la démarrer instantanément). En revanche, les centres de production utilisant des turbines à gaz peuvent rapidement être mobilisés mais à un coût de production plus élevé. Les énergies renouvelables (éolienne et photovoltaïque), quant à elles, se caractérisent par une production intermittente et peu prévisible liée à la présence effective de vent et de soleil respectivement.

14. Le transport de l'électricité est effectué sur le réseau de lignes à haute tension et la distribution sur le réseau de moyenne et basse tension. La circulation de l'électricité sur ces réseaux est contrainte par les capacités physiques des lignes et les règles de circulation avec un risque permanent de congestion sur certaines parties du réseau. Une partie de l'électricité est perdue pendant le transport du fait de la résistance des lignes. Ces pertes en ligne doivent être assumées collectivement. On retrouve donc à la fois des problèmes liés aux externalités 
de réseau et au bien public qu'il est difficile de résoudre avec les solutions traditionnelles. En effet, les conditions de circulation dans le réseau suivent les lois physiques de Kirchhoff qui obligent à une déconnexion entre les flux marchands et les flux physiques. Comme il n'est pas possible de "tracer" un flux électrique, les échanges physiques d'énergie ne correspondent pas forcément aux transactions commerciales entre les acheteurs et les vendeurs ce qui complique l'allocation des droits de propriété sur les flux d'électricité.

15. Il existe aussi plusieurs marchés de produits dérivés permettant aux entreprises de se couvrir contre les risques associés aux échanges sur les marchés de l'électricité. Ces marchés n'ont pas de spécificités reliées aux caractéristiques des produits qui servent de sous-jacents ; ils relèvent d'une logique financière pure. Nous n'en traiterons pas dans cet article.

16. Un smart market est programme de sélection des offres d'achat et/ou de vente qui maximise le surplus dans l'échange en respectant un ensemble de contraintes techniques propres au produit échangé.

17. En France, le législateur a défini les conditions pour permettre l'apparition de nouveaux acteurs sur les marchés de l'énergie : l'opérateur d'effacement dont la fonction consistera à agréger les effacements des consommateurs individuels en période de pointe quand les prix de l'énergie sont élevés et de valoriser ces effacements sur les marchés spot et d'ajustement (République Française [2013]).

18. Olson et al. [2003] conduisent une expérience avec un réseau de 9 nœuds.

19. Les centrales de pointe (aussi appelées centrales d'écrêtage de pointe) sont conçues pour aider à équilibrer les conditions énergétiques fluctuantes du réseau électrique. Il s'agit par exemple des centrales au gaz ou des turbine à combustion (TAC). Grâce à leur flexibilité et leur robustesse, elles sont capables d'apporter une réponse rapide à la demande fluctuante. 


\section{Références}

D. Ariely, A. Ockenfels et A. E. Roth [2005]: An Experimental Analysis of Ending Rules in Internet Auctions, The RAND Journal of Economics, 36(4), pp. 890-907.

S. Backerman, S. J. Rassenti et V. L. Smith [2000]: Efficiency and Income Shares in Highdemand Energy Networks: Who Receives the Congestion Rents When a Line Is Constrained?, Pacific Economic Review, 5(3), pp. 291-449.

J. Banks, M. Olson, D. Porter, S. J. Rassenti et V. L. Smith [2003]: Theory, Experiment and the Federal Communications Commission Spectrum Auctions, Journal of Economic Behavior and Organization, 51, pp. 303-50.

J. C. Bernard, T. Mount et W. Schulze [1998]: Alternative Auction Institutions for Electric Power Markets, Agricultural and Resource Economics Review, 27(2), pp. 125-31.

J. Brandts, S.S. Reynolds et A. Schram [2013]: Pivotal Suppliers and Market Power in Experimental Supply Function Competition, The Economic Journal, published online.

P. J. Brewer et C. R. Plott [1996]: A Binary Conflict Ascending Price (Bicap) Mechanism for the Decentralized Allocation of the Right to Use Railroad Tracks, International Journal of Industrial Organization, 14, pp. 857-87.

E. H. Chamberlin [1948]: An Experimental Imperfect Market, Journal of Political Economy, 56(April), pp. 95-108.

Y. Chen et T. Sönmez [2002]: Improving Efficiency of on-Campus Housing: An Experimental Study, The American Economic Review, 92(5), pp. 1669-86.

R. H. Coase [1960]: The Problem of Social Cost, Journal of Law and Economics, 3(ArticleType: research-article / Full publication date: Oct., 1960 / Copyright $\neg$ (C) 1960 The University of Chicago), pp. 1-44.

V. M. Coppinger, V. L. Smith et J. A. Titus [1980]: Incentives and Behavior in English, Dutch and Sealed-Bid Auctions, Economic Inquiry, 18(1), pp. 1-22.

J. C. Cox, B. Roberson et V. L. Smith [1982]: Theory and Behavior of Single Object Auctions, In Research in Experimental Economics, Vol. 2, ed. V. L. Smith, 1-43. Greenwich, Conn: JAI Press.

R. G. Cummings, C. A. Holt et S. K. Laury [2004]: Using Laboratory Experiments for Policymaking: An Example from the Georgia Irrigation Reduction Auction, Journal of Policy Analysis and Management, 23(2), pp. 341-63.

D. D. Davis et C. A. Holt [1993]: Experimental Economics, Princeton: Princeton University Press.

M.J. Denton, S. J. Rassenti et V. L. Smith [2001]: Spot Market Mechanism Design and Competitivity in Electric Power, Journal of Economic Behavior and Organization, 44, pp. 435-53.

D. Friedman [1993]: The Double Oral Auction Market Institution: A Survey, In The Continuous Double Auction Market: Institutions, Theories, and Evidence, ed. D. Friedman and J. Rust, 3-26. Reading Mass.: Addison-Wesley.

D. Friedman et J. Rust eds. The Double Auction Market Institutions, Theories, and Evidence. Menlo Park: Addison-Wesley Publishing Company, 1993.

D. Friedman et S. Sunder [1994]: Experimental Methods, In. Cambridge, U.K.: Cambridge University Press.

R. W. Hahn et M. V. V. Boening [1990]: An Experimental Examination of Spot Markets for Electricity, The Economic Journal, 100(403), pp. 1073-94. 
L. Hurwicz [1973]: The Design of Mechanisms for Resource Allocation, The American Economic Review, 63(2), pp. 1-30.

J. C. Jamison et C. R. Plott [1997]: Costly Offers and the Equilibration Properties of the Multiple Unit Continuous Double Auction under Conditions of Unpredictable Shifts of Demand and Supply, Journal of Economic Behavior and Organization, 32(April), pp. 591612.

C. Jullien, M. H. Khalfallah, V. Pignon, S. Robin et C. Staropoli [2009]: An Experimental Study of Investment Incentives Mechanisms in the Electricity Industry, In International Society for New Institutional Economics 13th Annual Conference. Berkeley.

C. Jullien, V. Pignon, S. Robin et C. Staropoli [2012]: Coordinating Cross-Border Congestion Management through Auctions: An Experimental Approach to European Solutions, Energy Economics, 34(1), pp. 1-13.

C. Jullien, S. Robin et C. Staropoli [2006]: Coordinating Cross-Border Congestion Management through Auctions: An Experimental Approach of European Solutions, In 5th Conference on Applied Infrastructure Research (INFRADAY). Berlin.

J. H. Kagel [1995]: Auctions: A Survey of Experimental Research, In Handbook of Experimental Economics, ed. J. H. Kagel and A. E. Roth, 501-85. Princeton: Princeton University Press.

P. Klemperer [2002]: What really matters in auction design, Journal of Economic Perspectives, Vol. 16, ${ }^{\circ} 1,169-189$.

R. Myerson, [1981]: Optimal Auction Design, Mathematics of Operations Research, 6, 5873.

C. Noussair, S. Robin et B. Ruffieux [1998]: The Effect of Transactions Costs on Continuous Double Auction Markets, Journal of Economic Behavior and Organization, 36(2), pp. 22133.

C. N. Noussair [2011]: Trends in Academic Publishing in Experimental Economics, In Wiley Online Conference on Communications with Economists: Current and Future Trends.

M. Olson, S. Rassenti, M. Rigdon et V. Smith [2003]: Market Design and Human Trading Behavior in Electricity Markets, IIE Transactions, 35(9), pp. 833-49.

C. R. Plott et P. Gray [1990]: The Multiple Unit Double Auction, Journal of Economic Behavior and Organization, 13(2), pp. pp 245-58.

S. Rassenti, S. Reynolds et V. Smit [1994]: Cotenancy and Competition in an Experimental Auction Market for Natural Gas Pipeline Networks, Economic Theory, 4(1), pp. 41-65.

S. J. Rassenti et V. L. Smith [1986]: Electric Utility Deregulation, Pricing Electric, Gas and Telecommunication Services,

S. J. Rassenti, V. L. Smith et R. Bulfin [1982]: A Combinatorial Auction Mechanism for Airport Time Slot Allocation, Bell Journal of Economics, 13, pp. 402-17.

S. J. Rassenti, V. L. Smith et B. J. Wilson [2002]: Using Experiments to Inform the Privatization/Deregulation Movement in Electricity, The Cato Journal, 21(3), pp. 515-44.

S. J. Rassenti, V. L. Smith et B. J. Wilson [2003]: Controlling Market Power and Price Spikes in Electricity Networks: Demand-Side Bidding, Proceedings of the National Academy of Sciences, 100(5), pp. 2998-3003.

République Française [2013]: Loi $N^{\circ}$ 2013-312 Du 15 Avril 2013 Visant À Préparer La Transition Vers Un Système Énergétique Sobre Et Portant Diverses Dispositions Sur La Tarification De L'eau Et Sur Les Éoliennes, In Journal Officiel. 
A. E. Roth [1995]: Introduction to Experimental Economics, in The Handbook of Experimental Economics, ed. J. H. Kagel and A. E. Roth, 3-109. Princeton, N.J.: Princeton University Press.

F. P. Sioshansi [2012]: Introduction, In Smart Grid: Integrating Renewable, Distributed \& Efficient Energy, ed. F. P. Sioshansi. Elsevier.

V. L. Smith [1962]: An Experimental Study of Competitive Market Behavior, Journal of Political Economy, 70(2), pp. 111-37.

V. L. Smith [1976]: Experimental Economics: Induced Value Theory, American Economic Review, 66(2), pp. 274-79.

V. L. Smith [1982a]: Markets as Economizers of Information: Experimental Examination of the 'Hayek Hypothesis', Economic Inquiry, 20, pp. 165-79.

V. L. Smith [1982b]: Microeconomic Systems as Experimental Science, American Economic Review, 72(5), pp. 923-55.

V. L. Smith [2003]: Constructivist and Ecological Rationality in Economics, The American Economic Review, 93(3), pp. 465-508.

W. Vickrey [1961]: Counterspeculation, Auction and Competitive Sealed Tenders, Journal of Finance, 16, pp. 8-37.

R. Wilson [2002]: Architecture of Power Markets, Econometrica, 70(4), pp. 1299-340. 